\title{
Genetic regulation of endothelial function
}

\author{
L C Jones, A D Hingorani
}

Heart 2005;91:1275-1277. doi: 10.1136/hrt.2005.061325

$\mathrm{T}$ he healthy vascular endothelium exerts atheroprotective actions through vasoactive mediators such as nitric oxide (NO), prostacyclin, and endothelium derived hyperpolarising factor (EDHF). There is evidence that as the endothelium ages, it is exposed to the damaging effects of raised blood pressure and increased concentrations of cholesterol, glucose, homocysteine, to products of the inflammatory response, and to the constituents of cigarette smoke, and these protective properties diminish leading to a state of endothelial dysfunction. ${ }^{1}$ Endothelial dysfunction can be detected in forearm or coronary arteries in vivo, before the development of clinical atherosclerosis, as an impairment of endothelium dependent agonist or flow mediated vasodilation. $^{2}$ Endothelial dysfunction by these methods correlates with cardiovascular risk factors, ${ }^{3}$ and may be predictive of incident cardiovascular events. ${ }^{4}$

Children with certain single gene disorders such as homocystinuria and familial hypercholesterolaemia, at risk of premature atherosclerosis, also exhibit early endothelial dysfunction. ${ }^{56}$ Although the more common forms of atherosclerosis manifest later in life, twin and adoption studies indicate that this more common form is also partly heritable, ${ }^{7}$ though inheritance is complex, arising as a result of common environmental exposures (risk factors) and many common gene variants (polymorphisms) with small to moderate effect. In common with other complex disorders, the causative genes have been difficult to identify. There have been few studies evaluating the heritability of endothelial function. However, vessels in the offspring or relatives of patients with premature cardiovascular disease show structural and functional changes more commonly, even before clinically manifest disease. ${ }^{8} 9$ Therefore, individual differences in endothelial function, and hence susceptibility to later atherosclerosis, might relate not only to different levels of exposure to risk factors but also to inter-individual differences in the carriage of risk alleles of genes expressed in the vascular endothelium. The atheroprotective actions of endothelial mediators such as NO suggest that genes that regulate synthesis and inactivation of these mediators might be important. Thus far, studies to address this have been small, and the findings are inconsistent probably because the genetic effect sizes in question are probably smaller then previously appreciated. There is now an increasing realisation that genetic studies, whether of intermediate phenotypes or of clinical outcomes, need to be much larger to provide reliable assessment of small but potentially important genetic effects. ${ }^{10}$ This article provides a brief overview of the most studied genes.

\section{VARIATION IN NITRIC OXIDE REGULATORY GENES, ENDOTHELIAL DYSFUNCTION, AND ATHEROSCLEROSIS}

Studies of normal NO synthesis suggest potential mechanisms to account for decreased NO availability that could underlie vascular disease. The factors and pathways of potential importance are illustrated in fig 1. Decreased NO bioavailability could result from reduced expression or activity of endothelial nitric oxide synthase (eNOS), increased generation of asymmetric dimethylarginine (ADMA; an endogenous circulating inhibitor of NOS), decreased availability of 6R-tetrahydrobiopterin $\left(\mathrm{BH}_{4} ;\right.$ an essential NOS co-factor), or increased inactivation of NO by reactive oxygen species (ROS) such as superoxide $\left(\mathrm{O}_{2}{ }^{-}\right){ }^{11}$

\section{GENETIC VARIATION IN NITRIC OXIDE REGULATORY GENES \\ Polymorphisms in the eNOS gene, NOS 3}

The NOS 3 gene has been the focus of intensive research to identify potentially functional polymorphisms or mutations that might influence expression or activity. To date more than 100 polymorphisms have been identified in, or in the vicinity of, the gene (NCBI SNP database, http// www.ncbi.nlm.nih.gov/SNP/).

More than 15 polymorphisms exist in the NOS3 promoter that might influence mRNA transcription and reduce gene expression. The $-786 \mathrm{~T} / \mathrm{C}$ promoter polymorphism influenced transcriptional activity in vitro in a luciferase/reporter assay system and was associated with coronary arterial spasm in Japanese subjects. ${ }^{12}$ In one study, endothelial cells from subjects with the CC genotype exhibited reduced shear stress induced eNOS mRNA transcription, and vascular rings from such subjects had diminished endothelium dependent vasodilation. ${ }^{13}$ However, the $-786 \mathrm{~T} / \mathrm{C}$ polymorphism has shown inconsistent associations with functional measures, and with clinical disease end points. A recent meta-analysis of studies involving 4882 cases and 9366 controls provided marginal evidence of increased risk among CC subjects (odds ratio $1.30,95 \%$ confidence interval (CI) 1.01 to 1.66 ; $\mathrm{p}=0.04) .^{14}$

Polymorphisms within the coding region of the NOS 3 gene could alter NOS enzymatic activity. One of the most studied eNOS polymorphisms $(894 \mathrm{G} \rightarrow \mathrm{T}$ ) within exon 7 is the only common polymorphism identified thus far that encodes an amino acid substitution-Glu298Asp (glutamate to aspartate at position 298). ${ }^{11}$ There is now a considerable debate as to whether this polymorphism is functional. Two studies have shown that eNOS Asp298 is subject to selective proteolytic cleavage in endothelial cells and vascular tissues that might account for reduced vascular NO generation. ${ }^{15}$ However, other studies suggest this finding might be artefactual. ${ }^{16}$ If this is the case, eNOS Asp298 might merely be a marker for another possibly functional variant elsewhere in the gene. Data from physiological studies have also been inconsistent. In some studies, individuals with the Asp298 allele exhibited altered vascular responses-for example, a reduced blood pressure fall following exercise training, a lower basal blood flow and reduced vasodilation to adenosine in the coronary

Abbreviations: ADMA, asymmetric dimethylarginine; $\mathrm{BH}_{4}$, tetrahydrobiopterin; DDAH, dimethylarginine dimethylaminohydrolases; EDHF, endothelium derived hyperpolarising factor; eNOS, endothelial nitric oxide synthase; FMD, flow mediated dilatation; LDL, low density lipoprotein; $\mathrm{NO}$, nitric oxide; $\mathrm{O}_{2}{ }^{-}$, superoxide; $\mathrm{ROS}$, reactive oxygen species 


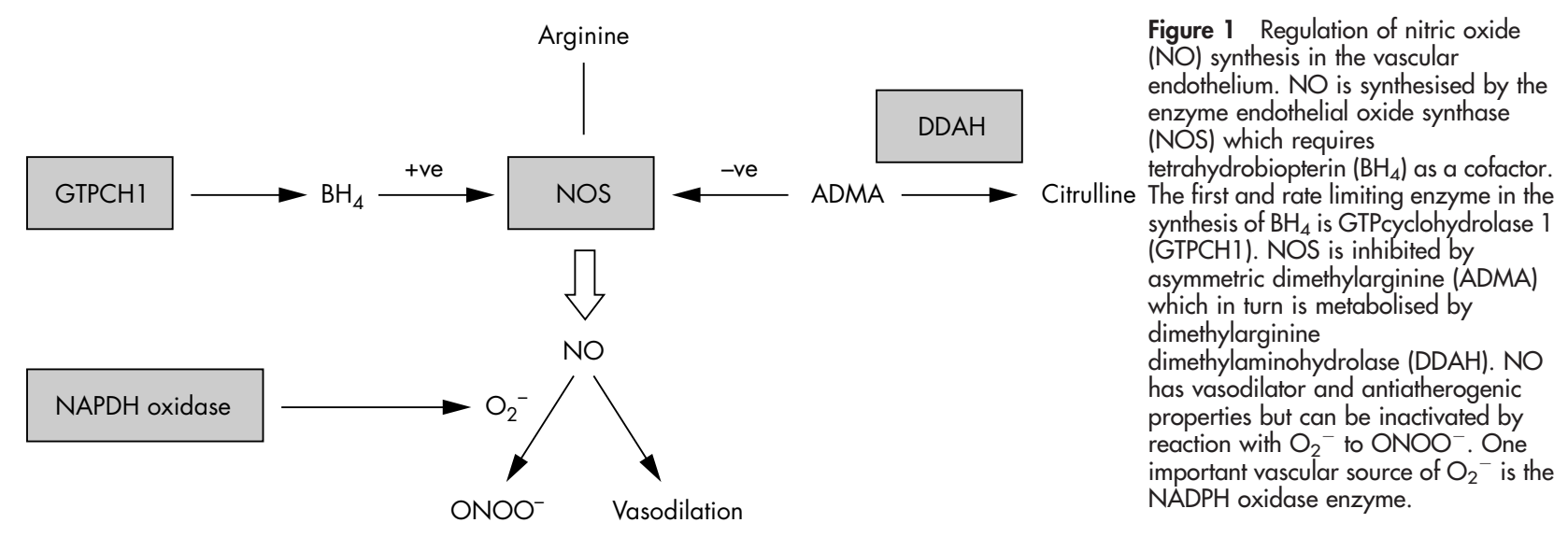

arteries, an enhanced systemic pressor response to phenylephrine, a reduced flow mediated dilatation (FMD) of the brachial artery if concurrent smokers, impaired dilator responses to acetylcholine if hypertensive, or impaired enhancement of FMD during the first trimester of pregnancy. ${ }^{17}$ However, not all studies are consistent with these findings. ${ }^{18}$ Finally, considering clinical outcomes rather then endothelial function as an end point, a meta-analysis of 6372 cases and 6591 controls identified an odds ratio for ischaemic heart disease of 1.33 (95\% CI 1.15 to $1.54 ; p=0.0001$ ) among individuals homozygous for eNOS Asp298. ${ }^{19}$ Given these inconsistencies, additional molecular studies are required, as are very large scale genetic association studies of endothelial function or clinical outcomes, to exclude the possibility that the inconsistent results of small scale physiological studies are not the result of random type I and type II error, and that meta-analyses are not severely affected by reporting bias.

\section{Polymorphisms in genes regulating ADMA}

Endogenous asymmetric methylarginines might have a profound effect on NO synthesis by inhibiting the NOS enzyme. ADMA, an analogue of the NOS substrate Larginine, is raised in various cardiovascular disease states, ${ }^{20}$ including hypertension, renal failure, and peripheral arterial occlusive disease. ADMA is removed from the circulation via metabolism by two isoforms of dimethylarginine dimethylaminohydrolases (DDAH), the DDAH2 isoform predominating in tissues expressing eNOS, such as the endothelium. The cause of raised ADMA in patients at risk of vascular disease is unknown, but common genetic variation within the DDAH enzymes that alter expression or activity might provide one potential explanation. Six polymorphisms have been identified in the DDAH2 gene. Five lie upstream of the translation start site and might influence gene transcription. An insertion deletion polymorphism $(6 \mathrm{G} / 7 \mathrm{G})$ at position -871 that lies with the basal promoter region influenced DDAH2 promoter activity in a promoter/reporter assay. ${ }^{21}$ Additional studies are required to clarify the effect of this variant on ADMA metabolism and endothelial function in vivo.

\section{Polymorphisms in genes regulating $\mathrm{BH} 4$}

Deficiency of the NOS cofactor, $\mathrm{BH}_{4}$, from reduced synthesis or increased oxidative inactivation could lead to reduced NO availability. When $\mathrm{BH}_{4}$ is limiting, NOS generates $\mathrm{O}_{2}{ }^{-}$rather than NO from oxygen and NADPH. ${ }^{22}$ The amount of available $\mathrm{BH}_{4}$ depends on rates of synthesis and inactivation. Inactivation of $\mathrm{BH}_{4}$ is probably through reaction with ROS generated within the vessel wall. The de novo production of $\mathrm{BH}_{4}$ is reliant upon three enzymes-GTPcyclohydrolase 1 , 6-pyruvoyl-tetrahydropterin synthase, and sepiapterin reductase. Local intravascular administration of $\mathrm{BH}_{4}$ to smokers, hypertensives, or hypercholesterolemics can reinstate normal endothelial function, whereas $\mathrm{BH}_{4}$ has no effect on healthy vessels at the same dose. ${ }^{22}$ This observation suggests that $\mathrm{BH}_{4}$ availability may be limited in diseased vessels. The initial step in the BH4 synthesis is the conversion of GTP to dihydroneopterin triphosphate by GTP cyclohydrolase 1 (GTPCH-1), which is rate-limiting. GTPCH-1 expression can be upregulated by cytokines and this is associated with elevation of intracellular $\mathrm{BH}_{4}$ in endothelial cells. More than 80 rare mutations have been reported in the human $\mathrm{GCH} l$ gene $\left(\mathrm{BH}_{4}\right.$ database, http://www.bh4.org), linked with the rare monogenic neurological disorder DOPA responsive dystonia. A recent study has identified two common polymorphisms in the promoter region of the gene that may be associated with plasma concentrations of neopterin (a marker of BH4 synthesis) (Jones LC et al, 2003, unpublished observations). There have been no published studies of variation in gene encoding downstream enzymes in the $\mathrm{BH}_{4}$ synthetic pathway, or of effects on endothelial function.

\section{Polymorphism in the gene encoding the $\mathrm{p} 22$ phox subunit of NADPH oxidase (CYB A)}

ROS mediate oxidation of low density lipoprotein (LDL), and promote smooth muscle cell growth, migration, and apoptosis, all of which occur during atherosclerosis. ${ }^{23} \mathrm{NO}$ also reacts rapidly with $\mathrm{O}_{2}{ }^{-}$to produce $\mathrm{ONOO}^{-}$, which can lead to protein and DNA damage, and results in "quenching" of the atheroprotective functions of NO. ROS may be elevated in disease states such as hypertension, heart failure, hypercholesterolaemia, and diabetes, possibly contributing to the endothelial dysfunction. Reduced inactivation of $\mathrm{O}_{2}{ }^{-}$, for example, by superoxide dismutase enzymes, might also contribute.

The vasculature is a site of $\mathrm{O}_{2}{ }^{-}$generation, ${ }^{23}$ through several enzymes including NADPH oxidases, and, under some circumstances, NOS itself. NADPH oxidase expression or activity may be upregulated in diseased blood vessels, and there has been interest in genetic variation in one particular subunit of this enzyme complex, p22phox, as a risk factor for vascular disease. Rare mutations of the p22phox gene (CYBA) cause autosomal recessive chronic granulomatous disease, but vascular function has not been systematically investigated in these individuals, and it is not known whether they are less atherosclerosis prone. Small scale studies of a common CYBA C242T polymorphism, that encodes a His72 $\rightarrow$ Tyr substitution in a potential heme binding site, have been conflicting. For example, the $242 \mathrm{~T}$ allele was associated with reduced NADPH oxidase activity in blood 
vessels from patients with coronary artery disease (CAD). ${ }^{24}$ Carriage of the $\mathrm{T}$ allele was also associated with augmented FMD of the brachial artery in 93 patients, 30\% of whom had evidence of $\mathrm{CAD}^{25}$ suggesting that this allele may exert a protective effect by down regulating $\mathrm{O}_{2}{ }^{-}$production. This was supported by association studies in Japanese subjects in which the 242T allele was present at higher frequency in control subjects than individuals with CAD or coronary spasm. ${ }^{26}{ }^{27}$ In contrast, the $242 \mathrm{~T}$ allele was associated with increased progression of $\mathrm{CAD}$ and decreased regression of disease upon lipid lowering treatment compared to CC homozygotes in a study of 313 individuals from the lipoprotein and coronary artery study. ${ }^{28}$ The C242T polymorphism was not associated with the occurrence or severity of CAD in a cohort of 689 Australian whites. ${ }^{29}$ Associations of the C242T variant with endothelial function have also been questioned. ${ }^{30}$ Some of these uncertainties might be resolved by systematic reviews and meta-analyses of the relevant genotype-disease association studies.

\section{OTHER POLYMORPHISMS INVESTIGATED FOR ASSOCIATIONS WITH ENDOTHELIAL FUNCTION}

Unsurprisingly polymorphisms in a variety of other genes whose products have been implicated in endothelial dysfunction have been tested for associations with endothelium dependent vasoreactivity. These include angiotensin converting enzyme, methylene tetrahydrofolate reductase, neuropeptide Y, the bradykinin receptor, interleukin-6, osteoprotogerin, glutathione cysteine ligase, and the Gprotein $\beta 3$-subunit. Results have either been inconsistent or data have been limited to single studies, and it has therefore been difficult to draw firm conclusions about these genes. There have also been rather few studies of genes that might regulate the availability other endothelial mediators such as endothelin-1, prostacyclin, and EDHF.

\section{FUTURE APPROACHES}

The appreciation that common gene variants may, at best, have small to modest effects on physiology and disease susceptibility has led to the understanding that future studies of complex disease susceptibility, whether they relate to clinical end points or intermediate phenotypes such as endothelial function, will need to be much larger in size. Meta-analysis to pool data from small underpowered studies with inconsistent findings may prove fruitful but there is also a need for larger definitive studies. Non-invasive assessment of vascular function by ultrasound using measures of FMD, pulse wave analysis, and arterial stiffness is now feasible in very large datasets numbering several thousand. The almost unique ability to gather such detailed phenotypic information in large numbers of human subjects which, in the case of FMD, may have independent prognostic significance, will in future offer the opportunity of defining genes that regulate vascular function, and will provide an insight into the interaction between genes and environmental exposures in the development of atherosclerosis.

\section{ACKNOWLEDGEMENTS}

$\mathrm{ADH}$ is a British Heart Foundation Senior Research Fellow

\section{Authors' affiliations \\ L C Jones, A D Hingorani, Centre for Clinical Pharmacology, British Heart Foundation Laboratories, Department of Medicine, University College London, London, UK}

Correspondence to: Dr Aroon D Hingorani, Centre for Clinical Pharmacology, BHF Laboratories, Department of Medicine, 5 University Street, London WCIE 6JJ, UK; a.hingorani@ucl.ac.uk

\section{REFERENCES}

1 Vallance $P$, Chan N. Endothelial function and nitric oxide: clinical relevance. Heart $2001 ; 85: 342-50$

2 Corretti MC, Anderson TJ, Benjamin EJ, et al for the International Brachial Artery Reactivity Task Force. Guidelines for the ultrasound assessment of endothelial-dependent flow-mediated vasodilation of the brachial artery: a report of the international brachial artery reactivity task force [erratum in: $J$ Am Coll Cardiol 2002;39:1082]. J Am Coll Cardiol 2002;39:257-65.

3 Chan NN, Colhoun HM, Vallance P. Cardiovascular risk factors as determinants of endothelium-dependent and endothelium-independent vascular reactivity in the general population. J Am Coll Cardiol 2001;38:1814-20

4 Perticone F, Ceravolo R, Pujia A, et al. Prognostic significance of endothelial dysfunction in hypertensive patients. Circulation 2001;104:191-6.

5 Celermajer DS, Sorensen K, Ryalls M, et al. Impaired endothelial function occurs in the systemic arteries of children with homozygous homocystinuria but not in their heterozygous parents. J Am Coll Cardiol 1993;22:854-8.

6 Celermajer DS, Sorensen KE, Gooch VM, et al. Non-invasive detection of endothelial dysfunction in children and adults at risk of atherosclerosis. Lancet 1992;340:1111-5

7 Marenberg ME, Risch N, Berkman LF, et al. Genetic susceptibility to death from coronary heart disease in a study of twins. N Engl J Med 1994;330:1041-6.

8 Gaeta G, De Michele M, Cuomo S, et al. Arterial abnormalities in the offspring of patients with premature myocardial infarction. N Engl J Med 2000;343:840-6

9 Clarkson P, Celermajor DS, Powe AJ, et al. Endothelium-dependent dilatation is impaired in young healthy subjects with a family history of premature coronary disease. Circulation 1997:96:3378-83

10 Hingorani A. Resolving inconsistency in the results of genetic association studies of cardiovascular disease. Clin Sci (Lond) 2004;107:251-3.

11 Hingorani AD. Polymorphisms in endothelial nitric oxide synthase and atherogenesis: John French Lecture 2000. Atherosclerosis 2001;154:521-7.

12 Nakayama $M$, Yasue $H$, Yoshimura $M$, et al. T-786 $\rightarrow$ C mutation in the $5^{\prime}$ flanking region of the endothelial nitric oxide synthase gene is associated with coronary spasm. Circulation 1999;99:2864-70.

13 Cattaruzza M, Guzik TJ, Slodowski W, et al. Shear stress insensitivity of endothelial nitric oxide synthase expression as a genetic risk factor for coronary heart disease. Circ Res 2004;95:841-7.

14 Casas JP, Hingorani AD, Humphries SE, et al. Do meta-analyses of association studies of endothelial nitric oxide synthase variants and ischemic heart disease provide conclusive answers? Circulation 2004;110:e305-6.

15 Persu A, Stoenoiu MS, Messiaen T, et al. Modifier effect of ENOS in autosomal dominant polycystic kidney disease. Hum Mol Genet 2002;11:229-41

16 McDonald DM, Alp NJ, Channon KM. Functional comparison of the endothelial nitric oxide synthase Glu298Asp polymorphic variants in human endothelial cells. Pharmacogenetics 2004;14:831-9.

17 Hingorani AD. Endothelial nitric oxide synthase polymorphisms and hypertension. Curr Hypertens Rep 2003;5:19-25.

18 Guzik TJ, Black E, West NE, et al. Relationship between the G894T polymorphism (Glu298Asp variant) in endothelialnitric oxide synthase and nitric oxide-mediated endothelial function in human atherosclerosis. Am J Med Genet 2001;100:130-7.

19 Casas JP, Bautista LE, Humphries S, et al. Endothelial nitric oxide synthase genotype and ischaemic heart disease: meta-analysis of 30 studies, involving 23,028 subjects. Circulation 2004:109:1359-65.

20 Vallance P, Leiper J. Cardiovascular biology of the asymmetric dimethylarginine:dimethylarginine dimethylaminohydrolase pathway. Arterioscler Thromb Vasc Biol 2004;24:1023-30.

21 Jones LC, Tran CTL, Leiper JM, et al. Common genetic variation in a basa promoter element alters DDAH2 expression in endothelial cells. Biochem Biophys Res Comm 2003;310:836-43.

22 Alp NJ, Channon KM. Regulation of endothelial nitric oxide synthase by tetrahydrobiopterin in vascular disease. Arterioscler Thromb Vasc Biol 2004; 24:413-20.

23 Griendling KK, Sorescu D, Ushio-Fukai M. NADPH oxidase. Role in cardiovascular biology and disease. Circulation Res 2000;86:494-501.

24 Guzik TJ, West NE, Black E, McDonald D, et al. Functional effect of the C242T polymorphism in the NAD(P)H oxidase p22phox gene on vascular superoxide production in atherosclerosis. Circulation 2000:102:1744-7.

25 Schachinger V, Britten MB, Dimmeler S, et al. NADH/NADPH oxidase p22 phox gene polymorphism is associated with improved coronary endothelial vasodilator function. Eur Heart J 2001;22:96-101.

26 Inove N, Kawashima S, Kanazawa K, et al. Polymorphism of the NADH/ NADPH oxidase p22 phox gene in patients with coronary artery disease. Circulation 1998;97:135-7.

27 Murase Y, Yamada Y, Hirashiki A, et al. Genetic risk and gene-environment interaction in coronary artery spasm in Japanese men and women. Eur Heart J 2004;25:970-7.

28 Cahilly C, Ballantyne CM, Lim DS, et al. A variant of p22(phox), involved in generation of reactive oxygen species in the vessel wall, is associated with progression of coronary atherosclerosis. Circ Res 2000;86:391-5.

29 Cai H, Duarte N, Wilcken DE, et al. NADH/NADPH oxidase p22 phox C242T polymorphism and coronary artery disease in the Australian population. Eur J Clin Invest 1999;29:744-8.

30 Schneider MP, Hilgers KF, Huang Y, et al. The C242T p22phox polymorphism and endothelium-dependent vasodilation in subjects with hypercholesterolaemia. Clin Sci (Lond) 2003;105:97-103. 